\title{
Pegada hídrica de suínos e o impacto de estratégias nutricionais
}

\author{
Julio C. P. Palhares ${ }^{1}$
}

${ }^{1}$ Embrapa Pecuária Sudeste. São Carlos, SP. E-mail: julio.palhares@embrapa.br (Autor correspondente)

\section{Palavras-chave:}

aminoácidos

dessedentação

fitase

fósforo

minerais orgânicos

\begin{abstract}
R E S U M O
Objetivou-se, neste estudo, calcular a pegada hídrica dos suínos abatidos no estado de Santa Catarina na década de 2001 a 2011 e avaliar o impacto de estratégias nutricionais no valor das pegadas azul e cinza. Testaram-se cinco estratégias nutricionais a fim de avaliar os impactos: T1, ração convencional; T2, ração com aminoácidos; T3, ração com fitase; T4, ração com minerais orgânicos e T5, ração com as tecnologias (T2, T3 e T4). Os valores das pegadas apresentaram comportamento crescente ao longo da década, resultado do aumento do número de animais abatidos. O cálculo para $\mathrm{T} 1$ apresentou os maiores valores de pegada e para $\mathrm{T} 5$, os menores. A porcentagem de redução foi de $18 \%$ entre esses tratamentos. O maior valor da pegada cinza foi verificado para ração convencional $\left(15.073 \mathrm{~m}^{3}\right.$ ano $\left.^{-1}\right)$ e o menor para estratégia com uso de fitase $\left(11.307 \mathrm{~m}^{3}\right.$ ano $\left.{ }^{-1}\right)$. Para cada litro de água utilizado foram gerados $179 \mathrm{kcal} \mathrm{em} \mathrm{T1,} 200 \mathrm{kcal} \mathrm{em} \mathrm{T2,} 193 \mathrm{kcal} \mathrm{em} \mathrm{T3,} 200 \mathrm{kcal} \mathrm{em} \mathrm{T4} \mathrm{e} 218 \mathrm{kcal}$ em T5. A macrorregião Oeste representou de 75 a 77\% do total da pegada para determinado ano e a Sul de 9 a $12 \%$. Os resultados deste estudo sustentam que o uso de estratégias nutricionais é uma prática conservacionista de uso da água reduzindo o valor das pegadas azul e cinza.
\end{abstract}

Key words: amino acids drinking water organic minerals phosphorus phytase

\section{Water footprint of swines and the impact of nutritional strategies}

\begin{abstract}
A B S T R A C T
The study aims to calculate the water footprint of swines slaughter in Santa Catarina State in the decade of 2001-2011, and to evaluate the impact of nutritional strategies in the value of blue and gray water footprint. Five different nutritional strategies were assessed: T1, conventional diet, T2, diet with amino acids, T3, diet with phytase, T4, diet with organic minerals, T5, diet with the technologies (T2, T3 and T4). The values of water footprint showed increasing pattern over the decade, due to an increase in the number of animals slaughtered. Conventional diet had the highest value and the diet with three strategies the lowest. The reduction was $18 \%$ among these treatments. The highest value to gray water footprint was found for conventional diet $\left(15,073 \mathrm{~m}^{3}\right.$ year $\left.^{-1}\right)$ and lowest for strategy with phytase $\left(11,307 \mathrm{~m}^{3}\right.$ year $\left.^{-1}\right)$. For each liter of water used $179 \mathrm{kcal}$ in T1, 200 $\mathrm{kcal}$ in T2, $193 \mathrm{kcal}$ in T3, $200 \mathrm{kcal}$ in T4, and $218 \mathrm{kcal}$ in T5 were generated. The macro-region West accounted for 75 to $77 \%$ of the total water footprint for a given year and the South macro-region for 9 to $12 \%$. The results support that the use of nutritional strategies provide a swine production more conservationist in water use, reducing their blue and grey water footprints.
\end{abstract}

\section{INTRODUÇÃo}

A produção de carnes (bovina, suína e aves) deverá aumentar em 10,9 milhões de toneladas até 2022, representando um acréscimo de $43,2 \%$ em relação à produção de carnes de 2012. O crescimento projetado para carne suína no período 2011/2012 a 2021/2022 é de 2,0\% ao ano, o que sinaliza um valor relativamente elevado pois consegue atender ao consumo doméstico e às exportações. As projeções do consumo são de $1,8 \%$ ao ano para o mesmo período (MAPA, 2012). Socialmente, a atividade suinícola também é de grande relevância, sobretudo na região Sul do país onde a base produtiva é a da mão de obra familiar; além disto, por ser a atividade grande consumidora de grãos, possibilita o desenvolvimento das cadeias de milho e soja.

Os índices econômicos e produtivos da suinocultura nacional atestam a competência, o impacto positivo do uso de conhecimentos e tecnologias e o valor econômico e social que esse setor ainda propiciará ao país porém a análise bidimensional (economia/sociedade) não é válida quando se almeja a sustentabilidade do país, tal como a preservação e conservação de seus recursos naturais. Portanto, é fundamental que os benefícios econômicos e sociais sejam avaliados em concomitância com as realidades ambientais.

Durante os últimos 20 anos os pesquisadores desenvolveram métricas para ajudar a caracterizar, mapear e acompanhar as questões ambientais no planeta. Os estudos têm destacado a incompatibilidade entre a disponibilidade hídrica e a demanda de água (Drastig et al., 2010; Hoekstra et al., 2012; Prochnow et al., 2012; Zoebl, 2006). As metodologias são fundamentais para avaliar o desempenho das atividades agropecuárias e relevantes para orientar produtores e consumidores em suas decisões. A avaliação da demanda hídrica é uma forma de geração deste tipo de informação que auxilia na tomada de 
decisão por produtos de menor impacto no uso da água e da terra (Jeswani \& Azapagic, 2011; Pfister et al., 2011). Vanham \& Bidoglio (2013) verificaram, em avaliação da pegada hídrica de produtos agrícolas nos países da Comunidade Europeia, que os produtos de origem animal representaram mais de $50 \%$ do valor total. As carnes com maior impacto no valor da pegada foram a bovina e a de porco.

Há falta de conhecimento das necessidades hídricas dos animais. Aumentar o conhecimento da utilização da água pelos diferentes sistemas de produção e desenvolver métodos padronizados para quantificação desta utilização, é a melhor forma, senão a única, para atingir o equilíbrio hídrico das produções (Girard, 2012). Embora a água seja um elemento fundamental na produção de suínos e sua deficiência prejudique o desempenho dos animais, a relação água/produção ainda é um assunto que recebe pouca atenção até que um problema ocorra, surpreendendo sobre o quão pouco se sabe sobre esta relação (Patience, 2012).

A pegada hídrica de um produto é definida como o volume de água consumido, direta e indiretamente, para produzir o produto. A principal vantagem do método frente a outras métricas é que ele calcula a água efetivamente consumida e não a água captada, além de inserir o cálculo das águas verde e cinza (Hoekstra et al., 2011).

A pegada hídrica é uma medida volumétrica que mostra o consumo de água doce no tempo e no espaço, fornecendo informações sobre como o recurso é alocado para diferentes fins. O volume alocado fornece informações referentes ao uso da água, mas não fornece informações sobre um problema de escassez e poluição na unidade hidrográfica na qual há a alocação (Gerbens-Leenes \& Hoekstra, 2012).

O cálculo da pegada hídrica e sua relação com o território geram informações com relevantes impactos sociais, ambientais e econômicos, necessários para implantação dos instrumentos de gestão contidos na Política Nacional de Recursos Hídricos e proporcionam impactos científicos de grande importância fortalecendo o tema produção animal e recursos hídricos, possibilitando o desenvolvimento de pesquisas nas áreas de eficiência hídrica, tecnologias de tratamento de resíduos, mitigação dos impactos ambientais e zootecnia de precisão. Tais pesquisas também darão, ao Brasil, uma posição de igualdade frente a grupos de pesquisa internacionais que têm realizado cálculos de pegada hídrica para as commodities agropecuárias brasileiras a partir de referenciais produtivos contestáveis ou que não traduzem a realidade nacional.

A avaliação do impacto é uma inovação frente aos estudos publicados até o momento para produtos animais haja vista que esses se restringem a calcular a pegada hídrica e não avaliam como conhecimentos e tecnologias podem reduzir o valor calculado. Segundo Palhares (2011) a utilização da metodologia de cálculo da pegada hídrica propicia a visualização quantitativa dos fluxos hídricos inerentes à produção; com isto, ações mitigatórias podem ser delineadas a fim de dar maior eficiência a esses fluxos.

A pesquisa foi conduzida com o objetivo de calcular a pegada hídrica dos suínos abatidos no estado de Santa Catarina e avaliar o impacto de estratégias nutricionais no valor das pegadas azul e cinza.

\section{Material e Métodos}

Para o cálculo das pegadas azul e cinza dos suínos abatidos no Estado de Santa Catarina na década de 2001-2011 utilizouse o método proposto por Chapagain \& Hoekstra (2003). O número de suínos abatidos com inspeção federal, estadual ou municipal, a partir da pesquisa trimestral de abate de animais do IBGE cujos resultados são apresentados por ano e por macrorregião do estado, segundo classificação do IBGE.

A pegada azul se refere ao consumo de água azul (superfícial e subterrânea). "Consumo" é entendido como a perda de água no espaço hidrográfico. Esta perda ocorre por evaporação, na incorporação ao produto, à água que retorna para outra unidade hidrográfica e à água que retorna em um período diferente (Mekonnen \& Hoekstra, 2010). No cálculo da pegada azul foram considerados somente os usos diretos de água na propriedade rural: dessedentação dos animais, lavagem e limpeza das instalações e quantidade de água no produto.

O cálculo da pegada azul é apresentado na Eq. 1 .

$$
\begin{aligned}
\mathrm{PA} & =\left(\mathrm{C}_{\mathrm{d}} \times 345 \times \mathrm{AB}\right)+\left(\mathrm{C}_{1} \times \mathrm{AB}\right)+ \\
& +\mathrm{Ap}[(\mathrm{AB} \times 0,115 \times 0,74 \times 0,66)+(\mathrm{AB} \times 0,04)]
\end{aligned}
$$

em que:

$\mathrm{PA}$ - pegada azul, $\mathrm{m}^{3}$ ano $^{-1}$

$\mathrm{C}_{\mathrm{d}}$ - consumo de água de dessedentação, para um alojamento de 345 dias

$\mathrm{C}_{1}$ - consumo de água de limpeza, 3,4 $\mathrm{L}_{\text {animal }}{ }^{-1}$, com lavagem somente na desocupação da instalação

$\mathrm{A}_{\mathrm{p}}$ quantidade de água no produto considerando-se uma média de 66\% de água por kg de carne (NEPA, 2011), fator de adição de $4 \%$ de água (água contida no sangue, glândulas, vísceras e conteúdo estomacal, entre outros.), rendimento de carcaça fria de $74 \%$ e peso ao abate de $115 \mathrm{~kg}$

$\mathrm{AB}$ - animais abatidos no ano ou na macrorregião

A pegada cinza é definida como o volume de água necessário para assimilar a carga de poluentes com base nas concentrações naturais e nos padrões legais. A concentração natural é aquela verificada quando não há atividade humana na área. Quando as concentrações naturais não são conhecidas, mas se estima ser baixa, seu valor pode ser assumido como sendo zero. Neste estudo referido valor foi assumido como zero.

O cálculo da pegada cinza é apresentado na Eq. 2.

$$
\mathrm{PC}=\frac{\alpha \times \text { Apl. }}{\mathrm{C}_{\max }}
$$

em que:

PC - pegada cinza, $\mathrm{m}^{3}$ ano $^{-1}$

a - fator fixo, para o fósforo total, sendo $a=0,11$ (Basso, 
Apl. - quantidade de dejeto suíno aplicado, $\mathrm{kg}$ de $\mathrm{P}_{\mathrm{ano}}{ }^{-1}$. Considerou-se a aplicação de $50 \mathrm{~m}^{3}$ de dejeto suíno aplicado por hectare por ano (Instrução Normativa $n^{\circ} 11$ da Fatma) e um dejeto com $2,5 \%$ de matéria seca

$\mathrm{C}_{\max }$ - concentração máxima permitida do elemento de acordo com a Resolução Conama $\mathrm{n}^{0} .357$, fósforo total $0,10 \mathrm{mg} \mathrm{L}^{-1}$

A escolha pelo elemento fósforo para o cálculo da pegada se deve à sua importância em processos de eutrofização dos corpos de água superficiais.

A atividade suinícola tem perfil difuso de poluição devido ao uso dos dejetos como fertilizante. Hoekstra et al. (2011) propõem a determinação de um fator adimensional fixo $(\alpha)$ que representa a fração de dejeto suíno escorrida superficialmente e que pode atingir o corpo d'água. $\mathrm{O}$ fator $a$ foi determinado com base no trabalho de Basso (2003) que desenvolveu um estudo com as seguintes características: área durante oito anos em plantio direto em Argissolo Vermelho Distrófico arênico com sucessão de culturas aveia preta/milho/nabo forrageiro, aplicados $40 \mathrm{~m}^{3} \mathrm{ha}^{-1}$ de dejeto líquido de suíno durante dois anos, distribuído a lanço e em superfície antes da semeadura de cada espécie na sucessão.

A última fase do método propõe a formulação de políticas e ações que podem ser conduzidas para reduzir a pegada. Sabe-se que a formulação da dieta impacta o consumo de água pelos animais e a excreção de elementos, essas premissas foram utilizadas para avaliar o impacto nos valores das pegadas azul e cinza. As estratégias foram selecionadas a partir do trabalho de Palhares et al. (2009). Os tratamentos avaliados e as respectivas médias de consumo de água por animal por dia e excreção de fósforo total por ciclo de produção foram:

T1 - Dieta com alto nível de proteína bruta, suplementação mínima de aminoácidos e sem a inclusão de fitase e minerais orgânicos, consumo médio, 5,0 $\mathrm{L}_{\text {animal }}{ }^{-1} \mathrm{~d}^{-1}$ e excreção de fósforo $11 \mathrm{~g} \mathrm{~kg}^{-1}$ de dejeto;

T2 - Dieta formulada a partir de T1, com redução do nível de proteína bruta mediante a suplementação de lisina, metionina, treonina e triptofano industriais, observando-se a proteína ideal de todos os aminoácidos essenciais, consumo médio, 4,4 $\mathrm{L}$ animal ${ }^{-1} \mathrm{~d}^{-1}$ e excreção de fósforo de $10 \mathrm{~g} \mathrm{~kg}^{-1}$ de dejeto;

T3 - Dieta formulada a partir de T1 porém com a inclusão de fitase e redução dos teores de cálcio e fósforo da dieta, consumo médio, 4,6 $\mathrm{L}_{\text {animal }}{ }^{-1} \mathrm{~d}^{-1}$ e excreção de fósforo de $8,2 \mathrm{~g} \mathrm{~kg}^{-1}$ de dejeto;

T4 - Dieta formulada a partir de T1 mas com a suplementação de $40 \%$ de minerais orgânicos ( $\mathrm{Cu}, \mathrm{Zn}$ e $\mathrm{Mn}$ ) e $50 \%$ minerais inorgânicos, consumo médio, $4,4 \mathrm{~L}_{\text {animal }}{ }^{-1} \mathrm{~d}^{-1}$ e excreção de fósforo de $10 \mathrm{~g} \mathrm{~kg}^{-1}$ de dejeto;

T5 - Dieta formulada a partir de T1 combinando, porém, os tratamentos $\mathrm{T} 2$, T3 e T4, consumo médio, $4,0 \mathrm{~L}_{\text {animal }}{ }^{-1} \mathrm{~d}^{-1}$ e excreção de fósforo de $8,5 \mathrm{~g} \mathrm{~kg}^{-1}$ de dejeto.

\section{Resultados e Discussão}

A pegada hídrica para cada estratégia nutricional durante a década de estudo é apresentada na Tabela 1. Observa-se, para
Tabela 1. Pegada hídrica dos suínos abatidos no estado de Santa Catarina por estratégia nutricional

\begin{tabular}{cccccc}
\hline \multirow{2}{*}{ Ano } & \multicolumn{5}{c}{ Pegada hídrica $\left(\mathbf{m}^{3}\right.$ ano $\left.^{-1}\right)$} \\
\cline { 2 - 6 } 2001 & T1 & T2 & T3 & T4 & T5 \\
2002 & 3.437 .235 & 3.159 .364 & 3.283 .696 & 3.159 .398 & 2.903 .443 \\
2003 & 3.487 .114 & 3.066 .596 & 3.187 .185 & 3.066 .629 & 2.818 .159 \\
2004 & 3.706 .826 & 3.307 .078 & 3.233 .469 & 3.111 .119 & 2.859 .059 \\
2005 & 4.047 .597 & 3.611 .063 & 3.753 .615 & 3.611 .096 & 3.318 .700 \\
2006 & 4.590 .604 & 4.095 .450 & 4.257 .543 & 4.095 .484 & 3.764 .007 \\
2007 & 4.588 .953 & 4.093 .978 & 4.256 .010 & 4.094 .011 & 3.762 .653 \\
2008 & 5.030 .224 & 4.487 .612 & 4.665 .523 & 4.487 .645 & 4.124 .530 \\
2009 & 5.121 .155 & 4.568 .726 & 4.749 .910 & 4.568 .760 & 4.199 .100 \\
2010 & 5.011 .776 & 4.471 .156 & 4.648 .403 & 4.471 .189 & 4.109 .401 \\
2011 & 5.108 .022 & 4.557 .011 & 4.737 .722 & 4.557 .044 & 4.188 .330 \\
MD $^{(1)}$ & 4.333 .704 & 3.866 .284 & 4.019 .131 & 3.866 .317 & 3.553 .329 \\
PR $^{(2)}$ & $100 \%$ & $89 \%$ & $93 \%$ & $89 \%$ & $82 \%$ \\
\hline
\end{tabular}

$M D^{(1)}$ Média decenal; $\mathrm{PR}^{(2)}$ Porcentagem de redução do valor da pegada em relação a $\mathrm{T1}$, considerando-se as médias decenais. T1 - Ração convencional; T2 - Ração com aminoácidos, T3Ração com fitase, T4 - Ração com minerais orgânicos; T5- Ração com as tecnologias T2, T3 e T4

todas as estratégias, que o valor da pegada hídrica apresenta variação crescente, resultado do aumento do número de animais abatidos ao longo dos anos. Os resultados demonstram que o referido processo tem impacto direto na demanda de água pela atividade. $\mathrm{O}$ aumento do número de cabeças deve ocorrer considerando-se a disponibilidade de recursos hídricos na unidade hidrográfica e na região e os outros usos.

A soma das pegadas azul e cinza totaliza $1.078 \mathrm{~L} \mathrm{~kg}^{-1}$. A pegada hídrica média global calculada por Mekonnen \& Hoekstra (2010) é $5.988 \mathrm{~L} \mathrm{~kg}^{-1}$ de carne suína, valor este muito acima dos valores calculados neste estudo. As razões para tais diferenças são: diferentes "fronteiras" de cálculo, diferentes sistemas de produção e manejo, consumos de água considerados para o cálculo da água azul e fontes de poluição e elementos poluentes utilizados no cálculo da água cinza. O método preconiza que os valores calculados não devem ser comparados uma vez que, além das diferentes premissas utilizadas em cada cálculo, a água possui uma identidade local, ou seja, o valor da pegada hídrica deve ser relacionado com a disponibilidade hídrica e qualidade da água na região de cálculo.

O cálculo para ração convencional apresentou os maiores valores de pegada hídrica e, para ração com as tecnologias T2, T3 e T4, os menores. Com base na porcentagem de redução, a média decenal foi $18 \%$ menor para $\mathrm{T} 5$ em relação a T1. A porcentagem mínima de redução foi de $7 \%$ entre T1 e T3. É provável que rações com maior tecnologia embarcada apresentam custo maior mas os cálculos demonstram o impacto positivo que a tecnologia tem na redução do valor da pegada hídrica. Hoekstra et al. (2011) atestam que, se existe uma tecnologia que propicie um valor menor da pegada hídrica, a pegada calculada é insustentável. Tomando essa premissa como base, pode-se afirmar que a pegada que considera a ração convencional é a mais insustentável e a que considera as tecnologias T2, T3 e T4 é a mais sustentável por utilizar menos água para produção do mesmo quilograma de carne.

A redução do consumo de água pelo animal por dia reduz o impacto da dessedentação no valor da pegada azul. Em T1 a dessedentação representou $90 \%$ do valor da pegada, $89 \%$ em T2, 
T3 e T4 e 88\% em T5. Assim, a porcentagem de água relativa ao produto foi maior para $\mathrm{T} 5(10,7 \%)$ e menor para $\mathrm{T} 1(9,5 \%)$ do valor da pegada azul.

O maior valor da pegada cinza foi verificado para ração convencional $\left(15.073 \mathrm{~m}^{3}\right.$ ano $\left.{ }^{-1}\right)$ em razão da mesma não fazer uso da fitase. A pegada hídrica com o uso de fitase apresentou o valor de $11.307 \mathrm{~m}^{3}$ ano $^{-1}$ e para T5 o valor intermediário de $11.715 \mathrm{~m}^{3}$ ano $^{-1}$. A redução da pegada cinza promoverá melhor relação suinocultura/conservação da qualidade das águas.

Considerando a relação litros por quilograma de carne magra (57\% do peso vivo) as relações foram: 9,8 para T1; 8,7 para T2; 9,1 para T3; 8,7 para T4; 8,0 $\mathrm{L} \mathrm{kg}^{-1}$ para T5. Um quilograma de carne fornece $1.750 \mathrm{kcal} \mathrm{kg}^{-1}$ (média dos cortes de bisteca, lombo e pernil) (NEPA, 2011). Para cada litro de água utilizado geraram-se 179 kcal em T1, $200 \mathrm{kcal} \mathrm{em} \mathrm{T2,} 193 \mathrm{kcal}$ em T3, $200 \mathrm{kcal} \mathrm{em} \mathrm{T4} \mathrm{e} 218 \mathrm{kcal}$ em T5. Demonstra-se que T5, além de ser mais conservacionista no uso da água, também exerce impacto positivo na nutrição humana visto que com o mesmo litro de água é gerada mais energia. Informações como essas também são úteis para avaliação ambiental das dietas pelos consumidores. Frequentemente, veem-se campanhas contra o consumo de proteína animal em virtude de serem mais intensivas no uso da água do que as proteínas vegetais. Dispondo de tecnologias de manejo que reduzem este uso, demonstra-se que o consumo da proteína animal pode ocorrer de forma ambientalmente menos impactante. Esta informação também será útil para as agroindústrias na escolha de produtos mais sustentáveis e elaboração de estratégias de marketing, principalmente, para exportação para mercados que exigem produtos com maior valor agregado.

Pesquisas econômicas devem ser conduzidas avaliando o impacto de cada estratégia nutricional no custo da água e no custo de produção da atividade a fim de identificar qual a melhor relação custo hídrico/custo de produção. Esta identificação auxiliará na tomada de decisão pelos atores e na promoção da sustentabilidade.

Na Tabela 2 se demonstra a economia de água de cada estratégia em relação ao valor da pegada de T1. Por ter a menor pegada, o T5 apresentou a maior economia de água de todas as estratégias. Na década o somatório da economia foi de $8.584 .123 \mathrm{~m}^{3}$. Este valor equivale a $83 \%$ da soma das duas maiores pegadas de $\mathrm{T} 1$ e representa $22 \%$ do somatório dos valores anuais para T5. A estratégia que utilizou somente a fitase, apresentou a menor economia de água na década; em contrapartida, a menor pegada cinza demonstrando que em regiões com intensos conflitos pelo uso quantitativo da água esta não seria a melhor estratégia mas em áreas com elevado grau de degradação qualitativa dos recursos hídricos seria uma estratégia a ser considerada.

Segundo o Censo demográfico de 2010, a população catarinense estava dividida em $84 \%$ na área urbana e $16 \%$ na área rural. Considerando um consumo per capta por dia de 190 L para a população urbana e $90 \mathrm{~L}$ para a população rural, a demanda hídrica anual seria de $364.031 .812 \mathrm{~m}^{3}$ ano${ }^{1}$ e $34.691 .817 \mathrm{~m}^{3} \mathrm{ano}^{-1}$, respectivamente. Relacionando as demandas com os valores da pegada hídrica para este ano,
Tabela 2. Economia de água em relação à ração convencional $\left(\mathrm{m}^{3}\right)$

\begin{tabular}{crrrr}
\hline Ano & T2 & \multicolumn{1}{c}{ T3 } & \multicolumn{1}{c}{ T4 } & \multicolumn{1}{c}{ T5 } \\
2001 & 381.871 & 257.540 & 381.838 & 637.792 \\
2002 & 370.644 & 250.055 & 370.611 & 619.081 \\
2003 & 376.029 & 253.645 & 375.995 & 628.055 \\
2004 & 399.747 & 269.457 & 399.714 & 667.586 \\
2005 & 436.535 & 293.982 & 436.501 & 728.898 \\
2006 & 495.154 & 333.062 & 495.121 & 826.597 \\
2007 & 494.976 & 332.943 & 494.942 & 826.300 \\
2008 & 542.612 & 364.701 & 542.579 & 905.694 \\
2009 & 552.428 & 371.245 & 552.395 & 922.055 \\
2010 & 540.621 & 363.373 & 540.587 & 902.375 \\
2011 & 551.011 & 370.300 & 550.978 & 919.692 \\
Economia na Década & 5.141 .627 & 3.460 .301 & 5.141 .262 & 8.584 .123 \\
\hline
\end{tabular}

*0s valores da tabela se referem à subtração do valor da pegada de T1 para cada valor de pegada dos outros tratamentos

T1 - Ração convencional; T2 - Ração com aminoácidos, T3 - Ração com fitase, T4 - Ração com minerais orgânicos; T5 - Ração com as tecnologias T2, T3 e T4

o valor de T1 representou 14\% da demanda rural, T2, T3 e T4, 13\% e T5 12\%. Da população total no ano de 2010, T1 representou $1,25 \%$ da demanda, T2 e T4, 1,12\%, T3, 1,16\% e T5 1,0\%. O impacto positivo das estratégias nutricionais no valor das pegadas sinaliza que uma eficiência hídrica melhor da suinocultura catarinense possibilitará aumento do conforto hídrico no estado. A melhoria da eficiência será ainda mais significativa considerando-se que a atividade está concentrada nas macrorregiões Oeste e Sul.

A Tabela 3 apresenta o cálculo da pegada hídrica por estratégia e por macrorregião. A macrorregião Oeste, devido à grande concentração de animais abatidos, representou de $75 \%$ a $77 \%$ do total da pegada hídrica para determinado ano; em seguida vem a macrorregião Sul, representando de 9 a 12\% do total calculado. A macrorregião da Grande Florianópolis, por ser caracterizada pela elevada densidade populacional e atividade industrial e com reduzido número de animais abatidos, apresentou a menor porcentagem do valor calculado, máximo de $0,7 \%$ do valor.

Somando os valores das pegadas na década excetuando a macrorregião Oeste, este somatório representa $31 \%$ do somatório da região demonstrando o elevado grau de concentração que apresenta a atividade no Estado. Esta situação, analisada em conjunto com a disponibilidade hídrica da macrorregião Oeste, os outros usos e as condições de qualidade das águas superficiais e subterrâneas podem significar uma situação de fragilidade hídrica.

Considerando apenas a pegada azul, a economia média de água na década promovida pelo uso da estratégia com as três tecnologias nutricionais é de $777.017 \mathrm{~m}^{3}$ valor que equivale a $92 \%$ da demanda média de água azul de um ano de todas as macrorregiões com exceção da Oeste. Analisando tão somente o impacto dessa estratégia na macrorregião Oeste, a economia média promovida equivale a $21 \%$ do valor da média decenal da pegada azul.

O cálculo das pegadas azul e cinza também poderá auxiliar nos processos de licenciamento das unidades produtivas, outorga de uso da água e fomentar políticas públicas e de zoneamento por explicitar a contabilidade hídrica da atividade e os impactos que tecnologias, manejos e práticas têm nesta contabilidade. 
Tabela 3. Pegada hídrica por macrorregião do estado de Santa Catarina

\begin{tabular}{|c|c|c|c|c|c|c|}
\hline \multirow[b]{2}{*}{ Ano } & \multicolumn{6}{|c|}{ T1/Macroregiôes $\left(m^{3}\right.$ ano $\left.^{-1}\right)$} \\
\hline & Oeste & Norte & Serrana & $\begin{array}{l}\text { Vale do } \\
\text { Itajaí }\end{array}$ & $\begin{array}{c}\text { Grande } \\
\text { Florianópolis }\end{array}$ & Sul \\
\hline 2001 & 2685979 & 134888 & 112533 & 170392 & 25141 & 412303 \\
\hline 2002 & 2638585 & 148676 & 105680 & 168722 & 24556 & 351021 \\
\hline 2003 & 2665958 & 160949 & 111281 & 181075 & 23143 & \\
\hline 2004 & 2859908 & 156951 & 95822 & 175542 & 24116 & 394487 \\
\hline 2005 & 3123312 & 161009 & 98270 & 215150 & 24777 & 425080 \\
\hline 2006 & 3521607 & 161217 & 97611 & 270975 & 22219 & 516975 \\
\hline 2007 & 3526892 & 182658 & 138593 & 308978 & 20314 & 411519 \\
\hline 2008 & 3816303 & 218663 & 154847 & 315783 & & \\
\hline 2009 & 3890296 & 226467 & 165583 & 325748 & 19203 & 493857 \\
\hline 2010 & 3811331 & 244672 & 170287 & 323326 & 19791 & 442368 \\
\hline & & & 171044 & 335629 & 14544 & 465637 \\
\hline \multicolumn{7}{|c|}{ T2/Macroregiões $\left(\mathrm{m}^{3} \mathrm{ano}^{-1}\right)$} \\
\hline 2001 & 335 & 120343 & 100398 & 152018 & 22430 & 67842 \\
\hline 2002 & 2354061 & 132644 & 94284 & 150528 & 21908 & 3170 \\
\hline 2003 & 2378478 & 143594 & 99281 & 161549 & & 07536 \\
\hline 2004 & 2551493 & 140026 & 85488 & 156612 & & \\
\hline 2005 & 2786462 & 143644 & 87672 & 191946 & 221 & 79235 \\
\hline 2006 & 3141757 & 143828 & 87083 & 241747 & & 1213 \\
\hline 2007 & 31464 & 162956 & 1236 & 2756 & & \\
\hline 2008 & 3404637 & 195076 & 138143 & 281 & & \\
\hline 2009 & 3470642 & 202037 & 1477 & 290 & & \\
\hline 2010 & 3400203 & 218279 & 151 & 2884 & & 4650 \\
\hline 2011 & 3433249 & 243362 & 152593 & 299424 & 12975 & 5408 \\
\hline \multicolumn{7}{|c|}{ T3/Macroregiões $\left(\mathrm{m}^{3}\right.$ ano $\left.^{-1}\right)$} \\
\hline 2001 & 490638 & 125079 & 104349 & 158000 & 23312 & 82318 \\
\hline 2002 & 244 & 137860 & & 156447 & & \\
\hline 2003 & 24720 & 149242 & 103187 & 167 & & \\
\hline 2004 & 2652015 & 145542 & 88856 & 162 & & 5811 \\
\hline 2005 & 2896462 & 149314 & & 199 & & 4206 \\
\hline 2006 & 3266104 & 149520 & 90529 & 251 & & 9467 \\
\hline 2007 & 3271004 & 169406 & & 286 & & 1662 \\
\hline 2008 & 3539 & 02810 & & 292 & & 7121 \\
\hline 2009 & 36082 & 210050 & 153 & 302 & & 58057 \\
\hline & 35349 & 226933 & & 299 & & \\
\hline & 35603 & & 158644 & 311298 & & \\
\hline \multicolumn{7}{|c|}{ T4/Macroregiões $\left(\mathrm{m}^{3}\right.$ ano $\left.^{-1}\right)$} \\
\hline 001 & & 1203 & 100399 & 152019 & 22430 & 67846 \\
\hline 2 & 235 & 132646 & 285 & 1505 & & 173 \\
\hline 2003 & 2378 & 1435 & & 161 & & \\
\hline 2004 & 2551 & 1400 & & 156 & & \\
\hline 2005 & 2786488 & 143645 & 87673 & 191 & & 79238 \\
\hline 2006 & & 1438 & & 241 & & \\
\hline 200 & 3146 & 162957 & & 275 & & \\
\hline 2008 & 3404662 & 195077 & 1381 & 281 & & 49312 \\
\hline & 3470667 & 202039 & & & & \\
\hline 2010 & 3400228 & 218281 & 151 & 2882 & & 4652 \\
\hline & & & 152594 & 299426 & 12975 & \\
\hline \multicolumn{7}{|c|}{ T5/Macroregiões $\left(m^{3}\right.$ ano $\left.0^{-1}\right)$} \\
\hline 2001 & 2202222 & 110594 & 92265 & 139704 & 20613 & 8045 \\
\hline & & 1218 & & & & \\
\hline 2003 & 2185800 & 1319 & & 148 & & \\
\hline 2004 & 2344849 & 128685 & & 143 & & 23441 \\
\hline 2005 & 2560861 & 132014 & & 176 & & \\
\hline 200 & $28874 \varsigma$ & 132188 & 80035 & 2221 & 18219 & 423887 \\
\hline 2007 & 2891829 & 149768 & 113638 & 2533 & & \\
\hline 200 & 3129176 & 179293 & & 258926 & & 412956 \\
\hline 20 & 3189855 & 1856 & & 267 & & \\
\hline 201 & 3125098 & 200619 & 139627 & 2651 & & \\
\hline 2011 & 3155485 & 223673 & 140248 & 275199 & 11926 & 381800 \\
\hline
\end{tabular}

T1 - Ração convencional; T2 - Ração com aminoácidos, T3 - Ração com fitase, T4 - Ração com minerais orgânicos; T5 - Ração com as tecnologias T2, T3 e T4

\section{Conclusões}

1. Os resultados deste estudo sustentam que as estratégias nutricionais propiciam que a atividade suinícola seja mais conservacionista no uso da água visto que reduzem o valor da pegada hídrica e os consumos de água azul e cinza.

2. A metodologia de cálculo permitiu identificar o impacto positivo das estratégias nutricionais demonstrando a redução do consumo de água azul e do potencial poluidor pelo uso dos resíduos como fertilizantes. Os resultados atestam que as informações geradas explicitam como o manejo nutricional pode promover a melhoria do desempenho hídrico.

\section{Agradecimentos}

Às contribuições técnicas e científicas dos pesquisadores Gustavo J. M. M. de Lima, Juliano C. Corrêa e Marcelo Miele, da Embrapa Suínos e Aves.

\section{Literatura Citada}

Basso, C. J. Perdas de nitrogênio e fósforo com aplicação no solo de dejetos líquidos de suínos. Santa Maria: UFSM, 2003. 125p. Tese Doutorado

Chapagain, A. K.; Hoekstra, A. Y. Virtual water flows between nations in relation to trade in livestock and livestock products. Netherlands: Unesco-IHE, 2003. 45p.

Drastig, K.; Prochnow, A.; Kraatz, S.; Klauss, H.; Plochl, M. Water footprint analysis for the assessment of milk production in Brandenburg (Germany). Advances Geosciense, v.27, p.6570. 2010.

Gerbens-Leenes, W.; Hoekstra, A.Y. The water footprint of sweeteners and bio-ethanol. Environment International, v.40, p.202-211, 2012.

Girard, C. L. Reducing the impact of animal production on the water supply: Increasing knowledge is the only solution. Animal Frontiers, v.2, p.1-2, 2012.

Hoekstra, A. Y.; Chapagain, A. K.; Aldaya, M. M.; Mekonnen, M. M. The water footprint assessment manual: Setting the global standard. London: Earthscan, 2011. 80p.

Hoekstra, A.Y.; Mekonnen, M. M.; Chapagain, A. K.; Mathews, R. E.; Richter, B. D. Global monthly water scarcity: Blue water footprints versus blue water availability. PlosOne, v.7, p.1-9, 2012.

Jeswani, H. K.; Azapagic, A. Water footprint: methodologies and a case study for assessing the impacts of water use. Journal of Cleaner Production, v.19, p.1288-1299, 2011.

MAPA - Ministério da Agricultura Pecuária e Abastecimento. Brasil projeções do agronegócio 2011/2012 a 2021/2022. Brasília: Ministério da Agricultura Pecuária e Abastecimento, 2012.50p.

Mekonnen, M. M.; Hoekstra, A. Y. The green, blue and grey water footprint of farm animals and animal products. Netherlands: Unesco-IHE, 2010. 50p.

NEPA - Núcleo De Estudos e Pesquisas em Alimentação. Tabela brasileira de composição de alimentos. em: http://www. unicamp.br/nepa/taco/. 25 Jun. 2012. 
Palhares, J. C. P. Pegada hídrica dos suínos abatidos nos Estados da Região Centro-Sul do Brasil. Acta Scientiarum Animal Sciences, v.33, p.309-314, 2011.

Palhares, J. C. P.; Gava, D.; Lima, G. J. M. M. de. Influência da estratégia nutricional sobre o consumo de água de suínos em crescimento e terminação. In: Simpósio Internacional sobre Gerenciamento de Resíduos de Animais, I. 2009, Florianópolis. Anais...Concórdia: SBERA, 2009. CD-Rom

Patience, J. F. The importance of water in pork production. Animal Frontiers, v.2, p.28-35, 2012.
Pfister, S.; Bayer, P.; Koehler, A.; Hellweg, S. Environmental impacts of water use in global crop production: Hotspots and trade-offs with land use. Environment. Science. Technology, v.45, p.5761-5768, 2011.

Prochnow, A.; Drastig, K.; Klauss, H.; Berg, W. Water use indicators at farm scale: methodology and case study. Food and Energy Security, v.1, p.29-46, 2012.

Vanham, D.; Bidoglio, G. A review on the indicator water footprint for the EU28. Ecological Indicators, v.26, p.61-75. 2013.

Zoebl, D. Is water productivity a useful concept in agricultural water management? Agricultural Water Management, v.84, p.265-273. 2006. 